\title{
Effect of Oral Spray on Dental Plaque Bacteria and Oral Epithelial Cells
}

\author{
Myoung-Hee Kim, Min Kyeng Lee, and Young Sun Hwang ${ }^{\dagger}$ \\ Department of Dental Hygiene, College of Health Science, Eulji University, Seongnam 13135, Korea
}

\begin{abstract}
Background: Good oral health is important for systemic body health and quality of life. Spray oral cleansers are increasingly preferred because of their convenience of carrying and the ease of oral hygiene management. In addition, many kinds of oral cleanser products containing various ingredients with antibacterial, washing, and moisturizing effects are being manufactured. However, concerns about the safety and side effects of oral sprays are increasing, and there is very little information regarding the use and care of oral sprays is available to consumers. This study aimed to investigate the effects of oral spray on oral bacteria and tissue to elucidate the factors that need to be considered when using oral sprays. Methods: The effects of oral spray on the growth of dental plaque bacteria was assessed using disk diffusion assays. Cytotoxicity and morphological changes in oral epithelial cells were observed by microscopy. The effects of oral spray on dental plaque growth were also confirmed on specimens from permanent incisors of bovines by Coomassie staining.

Results: The pH of spray products, such as Perioe Dental Cooling, Cool Sense, and Dentrix, were 3.65, 3.61, and 6.15, respectively. All tested spray products showed strong toxicity to dental plaque bacteria and oral epithelial cells. Compared with those on the control, dental plaque bacteria deposits on the enamel surface increased following the use of oral spray.

Conclusion: Three types of oral spray, namely Perioe Dental Cooling, Cool Sense, and Dentrix, strongly inhibited the growth of dental plaque bacteria and oral epithelial cells. The oral spray ingredient enhanced dental plaque growth on the enamel surface. Users should be informed of precautions when using oral sprays and the need for oral hygiene after its use.
\end{abstract}

Key Words: Bacteria, Cytotoxicity, Oral hygiene, Oral sprays, Plague

\section{Introduction}

Improvement in living standards has increased interest in overall systemic and oral health. From 2017, health insurance benefits have been expanded; the reduced medical expenses will reduce the burden to patients, which are expected to be more active in health activities to prevent disease along with increased examination and treatment of diseases ${ }^{1)}$. The demand for oral health and etiquette products such as functional toothpaste, mouthwash, toothbrush, tongue cleaner, and dental floss is growing ${ }^{2}$. In 2015, the global oral care market was worth about 45 trillion won and had grown at 5\% a year over the past five years. According to the Korea Trade-Investment Promotion
Agency (KOTRA), adult diapers and oral-related items were ranked among the predicted growth items in Japan in $2017^{3)}$. The market size growth of the nursing sponge brush to prevent aspiration pneumonia was particularly evident. Among products for oral health and hygiene, the growing interest in oral cleaning is increasing the demand for products with various purposes, including deodorization, oral cleaning, removal of biofilm, and oral moisturizing ${ }^{2)}$. In the case of spray-type oral cleansers, a variety of products are being released due to their convenience. However, there is a lack of awareness of problems such as oral dryness due to alcohol, oral diseases, and dental discoloration due to chloride compounds such as cetylpyridinium chloride (CPC). In addition, contamination 
of Toothette Oral Care Moisturizer (Advance Oral Moisturizer) was reported in October 2017. Therefore, the sales of products at online shopping malls and purchasing agency sites were suspended to prevent safety accidents ${ }^{4}$. Although there are differences in their compositions according to brand, they are widely classified as alcoholcontaining and non-alcoholic products as well as products containing propolis, xylitol, menthol, green tea extracts, and licorice extracts for the purpose of deodorization and cleaning. While the range of consumer choices has been expanded with the release of products containing a variety of functional ingredients, the manufacturers have provided use guidelines such as the application 'several times or three times a day directly into the mouth when necessary and you do not need to wash with water after use.' Studies are required on the safety of oral sprays and precautions in their use and the information should be provided to consumers. This study observed the effects of oral sprays on the growth of dental plaque bacteria and oral epithelial cells. In addition, the effects of oral sprays on dental plaque growth were also verified to determine the appropriate precautions for the use of these products.

\section{Materials and Methods}

\section{Materials}

A bovine permanent incisor with a healthy enamel surface was removed and stored in saline until use. Three kinds of oral sprays with high purchasing rates (Perioe Dental Cooling, Cool Sense, and Dentrix) were used for the experiments (Table 1). Distilled water was used as a control.

Table 1. Test Solutions

\begin{tabular}{|c|c|c|}
\hline Solution & Brand name & Manufacturer \\
\hline \multirow[t]{3}{*}{ Experiment } & $\begin{array}{l}\text { Perioe Dental } \\
\text { Cooling }\end{array}$ & $\begin{array}{l}\text { LG Household \& Health } \\
\text { Care (Seoul, Korea) }\end{array}$ \\
\hline & Cool Sense & $\begin{array}{l}\text { Docsmedi Inc. (Goyang, } \\
\text { Korea) }\end{array}$ \\
\hline & Dentrix & $\begin{array}{l}\text { Dongkook Pharmaceutical } \\
\text { Co., Ltd. (Seoul, Korea) }\end{array}$ \\
\hline $\begin{array}{l}\text { Control } \\
\text { (distilled water) }\end{array}$ & $\begin{array}{l}\text { Milli-Q Biocel } \\
\text { System }\end{array}$ & $\begin{array}{l}\text { Millipore (Darmstadt, } \\
\text { Germany) }\end{array}$ \\
\hline
\end{tabular}

2. Dental plaque bacteria culture and disc diffusion assay

Dental plaques from scaling were placed in sterilized Brain Heart Infusion (BHI) broth (Becton, Dickinson and Co., Baltimore, MD, USA) and cultivated in a $37^{\circ} \mathrm{C}$ shaking incubator ${ }^{5)}$. Dental plaque bacteria were then cultured in a BHI-agar broth (Becton, Dickinson and Co.) at around $\mathrm{OD}_{650}=1.5$. Oral spray solutions, ampicillin (10 $\mu \mathrm{g} / \mathrm{disc})$, and distilled water (10 $\mu \mathrm{l}$ each) were added to sterilized Whatman filter discs (Whatman, Maidstone, UK; $8 \mathrm{~mm}$ in diameter). After incubating for 16 hours, the clear zone around each filter disc was measured.

\section{Oral epithelial cell culture and microscopic analysis}

Oral epithelial cells were cultured in DMEM/F12 (3:1 ratio; Thermo Fisher Scientific Inc., Waltham, MA, USA) media at $37^{\circ} \mathrm{C}$ in a $\mathrm{CO}_{2}$ incubator ${ }^{6}$. Oral spray solutions were diluted with the same media for all experiments. The cellular morphology was observed by phase contrast microscopy (CKX41-A32PHP; Olympus, Tokyo, Japan).

\section{The $\mathrm{pH}$ analysis}

The $\mathrm{pH}$ of the oral sprays was measured repeatedly using a pH meter (Thermo Scientific Orion 2-Star Plus; Thermo Fisher Scientific Inc.) to calculate the mean value.

\section{Analysis of dental plaque formation}

Residual tissue and plaque were removed from the permanent incisor, and specimens measuring $5 \times 5 \mathrm{~mm}$ were prepared using a cutting $\operatorname{disc}^{7)}$. The specimens were randomly divided into four groups ( $\mathrm{n}=10$ in each group). The oral spray solutions were sprayed onto the enamel surface of each specimen and then put it into a broth with dental plaque bacteria. After a 12 hours shaking incubation, the specimens were washed three times with a phosphoric acid buffer ( $\mathrm{pH}$ 7.3) and stained with a Coomassie brilliant blue solution for 10 minutes. Images were obtained (COOLPIX A900; Nikon, Tokyo, Japan) and analyzed using ImageJ (National Institutes of Health, Bethesda, MD, USA).

\section{Statistical analysis}

The statistical analysis was performed using InStat ${ }^{\mathrm{TM}}$ 
statistical software (GraphPad Software Inc., San Diego, CA, USA). The statistical significance of the differences between groups was analyzed via repeated measures of one-way analysis of variance and Tukey's post-hoc analysis. p-values $<0.05$ were considered significant.

\section{Results}

\section{Effect of oral sprays on dental plaque bacteria}

To observe the effect of oral sprays on dental plaque bacteria, bacterial growth was observed by disc diffusion analysis. Ampicillin was used as a positive control. The same volumes of undiluted oral spray solutions were applied. Compared to the distilled water control, Perioe Dental Cooling, Cool Sense, and Dentrix all inhibited dental bacterial growth (Fig. 1). The diameters of the clear zones were $16.8 \pm 0.59 \mathrm{~mm}$ for Perioe Dental Cooling, $2.7 \pm 0.13 \mathrm{~mm}$ for Cool Sense, and $12.5 \pm 0.33 \mathrm{~mm}$ for Dentrix $(\mathrm{p}<0.01)$.
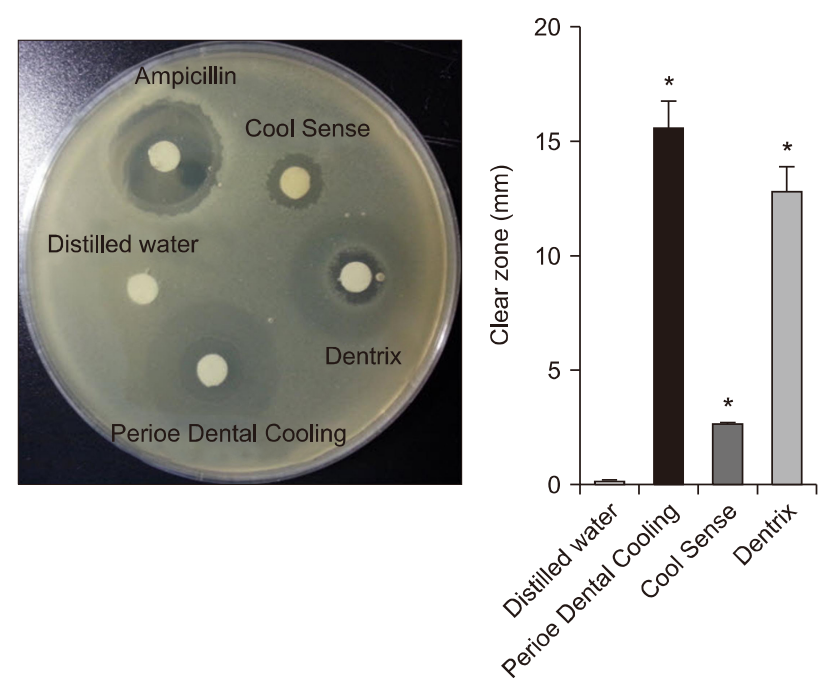

Fig. 1. Effect of oral sprays on the growth of dental plaque bacteria. Inhibitory effect of oral sprays $(10 \mu \mathrm{l}$ of stock solution/disc) on the growth of dental plaque bacteria. Perioe Dental Cooling, Cool Sense, and Dentrix were used as commercial oral spray. Ampicillin $(10 \mu \mathrm{g} /$ disc) was used positive control for assay. Distilled water was used for the control. Relative area of the growth inhibition (clear zone) of dental plaque bacteria was calculated with ImageJ (National Institutes of Health). Each values of individual experiments were expressed as a mean \pm standard error of three independent experiments. $* \mathrm{p}<0.01$ versus distilled water. Refer to Table 1 for the manufacturers' information.

\section{Effect of oral spray on gingival epithelial cells}

To observe the effect of oral sprays on gingival epithelial cells, cells were cultured in a medium containing the oral spray solution. Cytotoxicity was observed in mediums containing two- and five-fold diluted oral spray solutions. In the medium containing 10-fold diluted oral spray solutions, morphological changes and loss of cell attachment were observed compared to those in the control (Fig. 2). The cytotoxicity was particularly stronger for Perioe Dental Cooling. The morphological changes were observed about 10 minutes after oral spray addition and the number of floating cells also increased due to decreased cell adhesion.

\section{The $\mathrm{pH}$ of the oral sprays}

The $\mathrm{pH}$ of three oral sprays used in this experiment was measured after opening. The $\mathrm{pH}$ of Perioe Dental Cooling, Cool Sense, and Dentrix were 3.65, 3.61, and 6.15, respectively. No significant $\mathrm{pH}$ changes were observed in measurements performed 1 hour and 24 hours after opening.

\section{Effect of oral spray on dental plaque growth}

To test the effect of oral sprays on dental plaque growth, oral spray solutions were sprayed on the enamel surface five times and then dipped into a broth of dental plaque bacteria. After 12 hours shaking incubation, the specimens

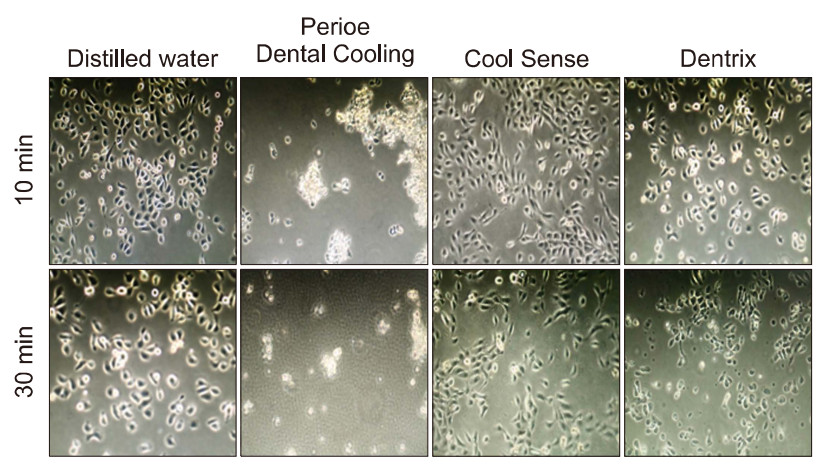

Fig. 2. Effect of oral sprays on morphology of gingival epithelial cells. The oral spray solution ( $1 / 10$ dilution in complete media) were treated in gingival epithelial cells for indicated time point (10 minutes and 30 minutes). The oral spray Perioe Dental Cooling, Cool Sense, and Dentrix were tested. Pictures were taken using a phase-contrast microscope at $200 \times$ magnifications. Distilled water was used for the control. Refer to Table 1 for the manufacturers' information. 

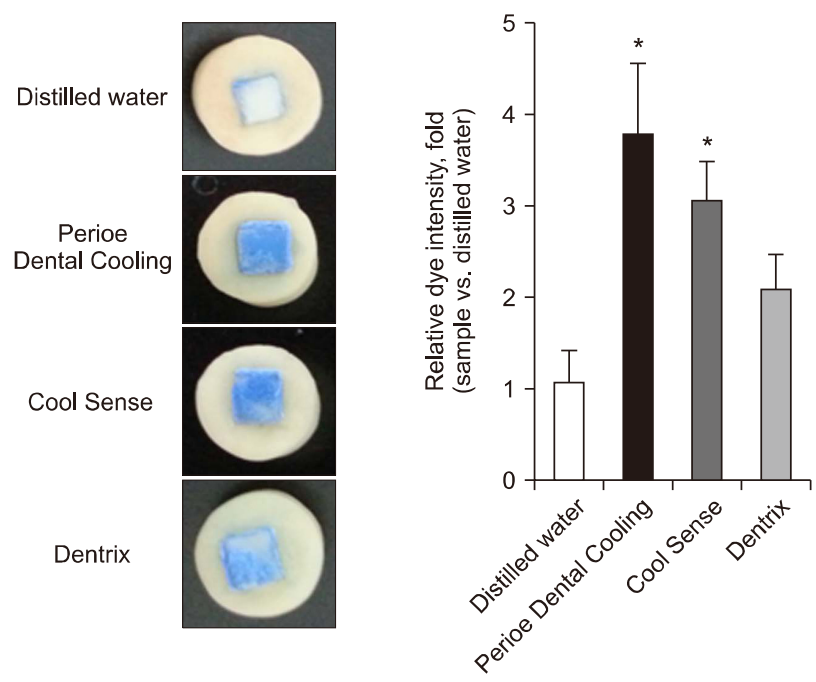

Fig. 3. Effect of oral sprays on adhesion of dental plaque bacteria to enamel surface. The bovine enamel specimens were applied with oral spray solution and were cultured in Brain Heart Infusion broth (Becton, Dickinson and Co.) with dental plaque bacteria. The bacteria adhere to specimens were stained with Coomassie brilliant blue G-250 dye. For density quantification, pictures were acquired and captured into ImageJ (National Institutes of Health) and were calculated in pixel intensities. Each values of individual experiments were expressed as a mean \pm standard error of three independent experiments. $\star p<0.01$ versus distilled water. Refer to Table 1 for the manufacturers' information.

were washed briefly and stained with Coomassie solution for 10 minutes. The degree of coloration of the residual plaque bacteria on the enamel surface was then analyzed. An increase in enamel surface coloring was observed in all test groups compared to that in the control. The increase in coloring for Dentrix compared to that for the control was not statistically significant, while those for Perioe Dental Cooling and Cool Sense were significant (Fig. 3).

\section{Discussion}

Oral sprays are used to conveniently correct halitosis. Although systemic disease, decreased saliva secretion, smoking, ovulation, and an empty stomach can cause halitosis, the most common causes are related to various factors in the oral cavity ${ }^{8}$. Protein compounds from oral epithelial cells, saliva, gingival crevicular fluid, blood, and residual food are metabolized mainly by gram-negative anaerobic oral bacteria, thereby producing volatile sulfides compounds, volatile nitrogen compounds, volatile fatty acids, acetone, and acetaldehyde, which induce halitosis ${ }^{9,10)}$. Despite the absence of bad breath, there may also be difficulties in interpersonal relations due to halitophobia. With the increase in social activities, the consumption of oral cleaning products for deodorization is further increasing. Poor oral hygiene, dental caries, periodontitis, xerostomia, and infectious disease such as oral candidiasis can cause halitosis. Therefore, the continued use of mouthwash products with antibacterial, cleaning, and moisturizing properties can be effective in reducing halitosis. Despite the increasing demand for spray-type mouthwash products with various functions, there is a lack of consumer awareness of the safety and side effects of these products and little information and caution regarding their use.

The main ingredients of oral sprays are sodium fluoride, CPC, menthol, methyl salicylate, etc., and some products contain alcohol. Sodium fluoride stabilizes the molecular structure of the dental apatite thereby increasing the acid resistance and preventing dental caries. Methyl salicylate maintains the scent of the product and acts as an antibacterial when hydrolyzed. Menthol and alcohol also act as antibacterial agents. Many studies suggest that oral cancer caused by alcohol remains controversial but serves as a risk factor ${ }^{11)}$. CPC is high in sterilization activity and is used as the main ingredient of mouthwashes, troches, and throat sprays. However, when the surfactant of the residual toothpaste reacts with $\mathrm{CPC}$, tooth discoloration may occur; therefore, it is necessary to check the product ingredients before use ${ }^{12)}$. In this study, we observed the effect of oral sprays on dental plaque bacteria and oral epithelial cells, thereby elucidating the cautions required for post-use of products and directions for oral hygiene management.

Three kinds of oral spray (Perioe Dental Cooling, Cool Sense, and Dentrix) with high purchasing rates were used in the experiment and their effects on dental plaque bacteria and oral epithelial cells were observed. Perioe Dental Cooling, Cool Sense, and Dentrix all showed antimicrobial activity and cytotoxicity to oral epithelial cells. Compared to Cool Sense and Dentrix, Perioe Dental Cooling showed strong cytotoxicity. This result indicates that the use of oral sprays is expected to reduce oral 
bacteria but that due to their strong cytotoxicity to oral epithelial cells, care is necessary for long-term use. The product information showed that Perioe Dental Cooling contains Curcima xanthorrhiza extract, fluorine, and ethanol, while Cool Sense contains purified water, propolis, xylitol, licorice extract, golden herb extract, peppermint extract, and hyaluronic acid. Dentrix contains CPC and a titrated extract of the unsaponifiable fraction of Zea mays L., ETIZM, which is used for the abatement of periodontal disease. Measurement of the $\mathrm{pH}$ of the oral sprays indicated a $\mathrm{pH}$ of 5.65 for Perioe Dental Cooling, pH 3.61 for Cool Sense, and pH 6.15 for Dentrix, which were significantly lower than the $\mathrm{pH}$ of 6.7 (rest) to 6.8 7.5 (stimulus) of whole saliva. Cytotoxicity might be involved the oral spray ingredients and their $\mathrm{pH}$.

We also observed the effect of oral sprays on dental plaque growth. The oral spray solutions were sprayed on the enamel surface and a specimen was incubated in a broth with dental plaque bacteria. Coomassie staining showed increased dental plaque bacteria growth on the enamel compared to that in the control. The effects of Perioe Dental Cooling and Cool Sense on dental plaque bacteria growth were statistically significant. The oral mucosa epithelium and dental surface are continuously washed by saliva in the mouth so significant cytotoxicity and dental plaque growth will not occur from the use of oral sprays. However, systemic disease or drugs that reduce saliva secretions may cause side effects on oral mucosa due to the continuous use of oral sprays. In addition, repeated use of oral sprays in poor oral hygiene can promote dental plaque growth; therefore, thorough oral hygiene management is required.

Dental plaque causes gingivitis and periodontitis, causing loss of alveolar bone. When glycoproteins in saliva attach to the enamel surface to form an acquired pellicle, oral bacteria form colonies on the surface ${ }^{13)}$. Streptococcus mutans secrete glucosyltransferase to produce highly adhesive, non-hydrous glucans that further enhance the adhesion of oral bacteria to dental plaque ${ }^{14)}$. The lipopolysaccharides of dental plaque bacteria in the periodontal pocket cause inflammation and can lead to periodontitis ${ }^{15)}$. Altogether, gram-negative anaerobic bacteria accelerate tissue damage by secreting cytolytic toxins such as epitheliotoxin; endotoxin; leukotoxin; and proteolytic enzymes such as collagenase, gelatinase, and elastase. The study results suggest that the continuous use of oral sprays without oral hygiene management can promote oral disease due to the growth of residual dental plaque. Therefore, oral hygiene management is required after the use of oral sprays and information on precautions for use should be provided to consumers.

\section{Notes}

\section{Conflict of interest}

No potential conflict of interest relevant to this article was reported.

\section{Ethical approval}

No ethical approval was needed for the study. There is no formal restriction on the collection of bovine teeth for research because the slaughter has been approved by the Animal and Plant Quarantine Agency.

\section{ORCID}

Myoung-Hee Kim, https://orcid.org/0000-0003-1589-4038

Min Kyeng Lee, https://orcid.org/0000-0002-8291-5380

Young Sun Hwang, https://orcid.org/0000-0001-7012-3434

\section{Acknowledgements}

This research was supported by Basic Science Research Program through the National Research Foundation of Korea (NRF) funded by the Ministry of Education, Science and Technology (2018R1D1A1B07042035).

\section{References}

1. National Health Insurance Service: Strengthening health insurance coverage. Retrieved July 4, 2018, from http:// medicare1.nhis.or.kr/hongbo/static/html/minisite/index.html (2017, August 9).

2. LG Household \& Health Care Ltd: Attracting 45 trillion won oral care market. Retrieved July 4, 2018, from http://newslabit. hankyung.com/news/app/newsview.php?aid=2016110127391 (2016, July 4).

3. Korea Trade-Investment Promotion Agency (KOTRA): 
Japan-Growing commodity items selected by super purchaser in 2017. Retrieved July 1, 2018, from http://news.kotra. or.kr/user/globalAllBbs/kotranews/album/2/globalBbsDataA llView.do?dataIdx $=156987 \&$ column=title \&search=\&search AreaCd $=10002 \&$ searchNationCd $=\&$ search TradeCd $=\&$ searc $\mathrm{hStartDate}=\&$ searchEndDate $=\&$ searchCategoryIdxs $=\&$ searc hIndustryCateIdx $=\&$ page $=5 \&$ row $=60(2017$, January 31$)$.

4. Korea Consumer Agency: Consumer Injury Surveillance System. Toothette Oral Care-Discontinued sales of some advance oral moisturizer spray products. Retrieved July 1, 2018, from https://www.ciss.go.kr/www/selectBbsNttView. do? key $=97 \&$ bbsNo $=81 \&$ nttNo $=31765 \&$ searchCtgry $=\&$ sear chCnd=all\&searchKrwd=\&pageIndex=1\&pageUnit=10\&int egrDeptCode $=(2017$, December 26).

5. Lee J, Nho YH, Yun SK, Hwang YS: Use of ethanol extracts of Terminalia chebula to prevent periodontal disease induced by dental plaque bacteria. BMC Complement Altern Med 17: 113, 2017. https://doi.org/10.1186/s12906-017-1619-1

6. Jung I, Park JH, Lee MK, Hwang YS: The cytotoxic effect of oral wet wipes on gingival cells. J Dent Hyg Sci 18: 76-84, 2018. https://doi.org/10.17135/jdhs.2018.18.2.76

7. Lim DS, Ban YH, Min YE, et al.: The effect of carbonated water on bovine enamel erosion and plaque adhesion. J Dent Hyg Sci 15: 437-444, 2015.

https://doi.org/10.17135/jdhs.2015.15.4.437

8. Porter SR, Scully C: Oral malodour (halitosis). BMJ 333: 632-
635, 2006. https://doi.org/10.1136/bmj.38954.631968.AE

9. Ramsay EI, Rao S, Madathil L, et al.: Honey in oral health and care: a mini review. J Oral Biosci 61: 32-36, 2019. https://doi.org/10.1016/j.job.2018.12.003

10. Kim KE, Han JH: Correlation among halitosis, xerostomia and stress in adults. J Dent Hyg Sci 16: 370-377, 2016. https://doi.org/10.17135/jdhs.2016.16.5.370

11. Bagnardi V, Rota M, Botteri E, et al.: Alcohol consumption and site-specific cancer risk: a comprehensive dose-response meta-analysis. Br J Cancer 112: 580-593, 2015. https://doi.org/10.1038/bjc.2014.579

12. Teng F, He T, Huang S, et al.: Cetylpyridinium chloride mouth rinses alleviate experimental gingivitis by inhibiting dental plaque maturation. Int J Oral Sci 8: 182-190, 2016. https://doi.org/10.1038/ijos.2016.18

13. Hannig C, Hannig M, Attin T: Enzymes in the acquired enamel pellicle. Eur J Oral Sci 113: 2-13, 2005. https://doi.org/10.1111/j.1600-0722.2004.00180.x

14. Lapirattanakul J, Takashima Y, Tantivitayakul $\mathrm{P}$, et al.: Cariogenic properties of Streptococcus mutans clinical isolates with sortase defects. Arch Oral Biol 81: 7-14, 2017. https://doi.org/10.1016/j.archoralbio.2017.04.018

15. Shaddox LM, Gonçalves PF, Vovk A, et al.: LPS-induced inflammatory response after therapy of aggressive periodontitis. J Dent Res 92: 702-708, 2013. https://doi.org/10.1177/0022034513495242 